\title{
Survival and Treatment Responses of Hepatic Arterial Infusion Chemotherapy for Advanced Hepatocellular Carcinoma
}

Jae Hyuk Choi, Woo Jin Chung*, Byung Kuk Jang and Jae Seok Hwang

Department of Internal Medicine, Keimyung University School of Medicine, Daegu, Republic of Korea

\begin{abstract}
Patients with advanced hepatocellular carcinoma (HCC) have a poor prognosis. Their median survival time was reported to be 2.7-4 months if they were left untreated. Hepatic arterial infusion chemotherapy (HAIC) has been regarded as one of the effective therapeutic modalities. So, the aim of this study was to evaluate survival and therapeutic responses of HAIC for advanced HCC. From January 2004 to November 2011, we retrospectively reviewed the data of patients with advanced HCC who received 2 sessions of HAIC or more. Tumor response thereto was measured by the abdominal CT scan at each session of HAIC using Modified Response Evaluation Criteria in Solid Tumors. 54 patients were enrolled. Their mean age was 57 year-old and 47 patients were male. 33 patients were under Child-Turcotte-Pugh Class A, 17 were under Class B, and 4 were under Class $\mathrm{C}$. The causes of HCC were HBV (70.3\%), HCV (11.1\%), and alcoholics (13.0\%). On average, patients received 4.2 sessions of HAIC. At the time of completing 2 sessions of HAIC, the median survival time of patients who achieved complete response (CR) or partial response (PR) (Group A) was 190 days, while that of patients who had stable disease (SD) or progressive disease (PD) (Group B) was 96 days $(p=0.265)$. At the time of completing 4 sessions of HAIC, the median survival time of Group A and Group B was 302 days and 111 days, respectively $(p=0.009)$. Advanced HCC without potal vein tumor thrombosis (PVTT) showed better therapeutic responses than advanced HCC with PVTT $(p=0.021)$. HAIC can be a useful therapeutic modality for patients with advanced HCC. Presence of PVTT can be an independent predictive factor for therapeutic response of HAIC. However, prospective studies for identifying predictive factors of better prognosis of advanced $\mathrm{HCC}$ are still needed.
\end{abstract}

Keywords: Hepatic arterial infusion chemotherapy (HAIC); Advanced hepatocelluar carcinoma; Hepatocellular carcinoma

\section{Introduction}

Even though the incidence rate of hepatocellular carcinoma (HCC) in Korea has recently decreased from 47 cases in 100,000 people in 1990 's to 39 cases in 100,000 people in 2000's, Korea has still a high prevalence of HCC, compared with other countries except Asia-Pacific region $[1,2]$.

Hepatocellular carcinoma is known to be the major- cause of death of patients with liver cirrhosis $[3,4]$. There are a number of treatment options including surgical interventions such as hepatic resection, liver transplantation, and non-surgical interventions such as the transcatheter arterial chemoembolization (TACE), the regional hepatic artery infusion chemotherapy (HAIC), the percutaneous ethanol injection, the high frequency diathermy. However, the prognosis of HCC remains unsatisfactory.

Surgical hepatic resection or liver transplantation is limited due to impaired liver function or involved hepatic failure, and TACE is infeasible for patients with portal vein tumor thrombosis due to the hepatic failure risk [5]. Systemic cancer chemotherapy has also failed to report satisfactory outcome [6]. Furthermore, high recurrent rates in HCC have been reported even after curative treatment including surgical hepatic resection [7], and most of recurrent cases progress to advanced HCC resulting in the extremely low survival rate in patients with portal vein tumor thrombosis or systemic metastasis $[8,9]$.

In previous studies, it was reported that the survival period of patients with HCC accompanied with portal vein tumor thrombosis was 1-2 months in the case that they did not receive effective treatments $[8,10,11]$. So, any effective treatment is keenly required to maintain the quality of life of patients with advanced HCC. Currently, TACE, systemic cancer chemotherapy, radiotherapy or HAIC are undergone for patients with HCC. Among these treatment methods, the regional HAIC is reported to increase the anti-cancerous effect and to decrease systemic adverse reactions by localizing chemotherapeutic agents within liver [12-17].

In the case that patients have HCC accompanied with portal vein tumor thrombosis (PVTT) or systemic metastasis, according to Barcelona Clinic of Liver Cancer (BCLC) treatment guideline, Sorafenib is recommendable as the standard therapy [18]. However, expected extension of survival rate among Asians is judged to be unsatisfactory, and its cost has also been considered as an actual major issue [18-20] in terms of the economical burden.

The regional HAIC is to enhance the regional therapeutic effect and decrease adverse reactions, compared with the systemic chemotherapy. And it has been reported to be effective in patients with advanced HCC who have a difficulty in using other conventional curative treatments have no therapeutic response thereto [21-25]. Therefore, the authors intended to analyze clinical outcomes of the regional HAIC in patients with advanced HCC, examine their survival rate and therapeutic effect, and analyze the predictor of response for the regional HAIC.

*Corresponding author: Woo Jin Chung, Department of Internal Medicine Keimyung University School of Medicine, 56 dalsung-ro, jung-gu, Daegu, Korea 700-712, Tel: (053) 250-7413; Fax: (053) 250-7088; E-mail: chung50@dsmc.or.kr

Received August 03, 2012; Accepted August 23, 2012; Published August 25, 2012

Citation: Choi JH, Chung WJ, Jang BK, Hwang JS (2012) Survival and Treatment Responses of Hepatic Arterial Infusion Chemotherapy for Advanced Hepatocellular Carcinoma. J Cancer Sci Ther 4: 292-298. doi:10.4172/1948-5956.1000157

Copyright: (c) 2012 Choi JH, et al. This is an open-access article distributed under the terms of the Creative Commons Attribution License, which permits unrestricted use, distribution, and reproduction in any medium, provided the original author and source are credited. 


\section{Materials and Methods}

\section{Material}

This study was conducted by reviewing medical records of patients who had treated with the regional HAIC after their cases were diagnosed as HCC between April 2004 and October 2011. The diagnosis of HCC was re-evaluated on the basis of the hepatocellular carcinoma treatment guideline suggested by the Korean liver cancer study group [26].

The regional HAIC was used to treat patients with advanced HCC that was defined as HCC for which surgical interventions or nonsurgical local procedures were unavailable or contraindicated [12$17,21-25,27-29]$

Accordingly, this study included patients with HCC without extrahepatic metastasis but having a tumor which was big enough to occupy most area of one lobe, patients for whom surgical operations or non-surgical local procedures such as high-frequency diathermy, percutaneous ethanol injection or TACE were unavailable or contraindicated due to PVTT and presence of the newly advanced tumor was identified, and patients who were unable to receive other local treatments due to locations and shapes of tumors recurring as multiple intrahepatic tumors after the surgical excision thereof.

Also, this study included patients who had at least 2 cycles of treatment and were followed up for 1 month or more so that their therapeutic responses could be evaluated by the abdominal CT image.

\section{Methods}

For the drug infusion, the hepatic artery was catheterized through the right or left femoral artery and the injection port was implanted at the subcutaneous pocket in the right or the left inguinal region. 54 patients who met the above criteria were repetitively treated for 5 days at intervals of 3 weeks with 5-FU (250 mg/day, day 1-5), cisplatin (10 $\mathrm{mg}$ /day, day 1-5), leucovorin (12 mg/day, day 1-5).

For following up, basically, serum alpha-fetoprotein (AFP, RIAKEY. One Step AFP IRMA Tube. ShinJin Medics, South Korea. Normal range $<15 \mathrm{ng} / \mathrm{mL}$ ) and the abdominal CT scan were performed every 2 cycles to evaluate therapeutic responses, and these test were also performed as required when there was a change in the patient state, such as sudden deterioration of liver function or an abdominal pain.

Serum AFP was evaluated by measuring the changes in the range over $200 \mathrm{ng} / \mathrm{mL}$ according to the practice guidelines for management of hepatocellular carcinoma of the Korean Liver Cancer Study Group. Serum AFP is also utilized as a 'confirmatory test' to discriminate HCC from other solid lesions of the liver. The levels of $200 \mathrm{ng} / \mathrm{mL}$ are commonly utilized for this purpose [26,30].

\section{Evaluation of therapeutic responses}

The abdominal CT image was followed up after 2 cycles of treatment so that their therapeutic responses could be evaluated. Treatment responses were evaluated by measuring the changes in the diameter of enhanced tumors on the abdominal CT image utilizing modified Response Evaluation Criteria in Solid Tumors (mRECIST) [31].

Complete response was defined as complete disappearance of the arteriogram of the lesion. Partial response was defined as the case that the major axis of the lesion was decreased $30 \%$ or more. Progressive disease was defined as the case that the major axis was increased $20 \%$ or more. Other state was defined as stable disease [32].

\section{Statistics}

Patient's characteristics (age and sex), causative liver disease, liver function index (Child-Turcotte-Pugh score, PT, Albumin, and Bilirubin), hematologic test (ALT, AFP), the characteristics of HCC (imaging and the presence and grade of PVTT by the criteria of the Liver Cancer Study Group of Japan) [33], the presence of ascites and treatment details were recorded for analysis thereof, and adverse reactions and complications were also recorded.

Chi-square test, Student's t-test and Fisher's exact test were used for statistical analysis, and Kaplan-Meier survival method was used to analyze the survival period of patients. All the results were expressed as median values or means \pm standard deviations. SSPS Version 18.0 (SPSS Inc., Chicago, IL, USA) was used for statistical analysis.

\section{Results and analysis}

Clinical outcomes were analyzed by reviewing medical records, and patients were traced to their death or the date of their last visit to hospital. Patients were individually contacted on the phone to confirm their survival and their survival period after the date of their last visit to hospital in order to analyze the survival period.

Complication was defined as an occurrence of new symptoms or changes in the treatment method during the follow-up period. Major complications included bleeding esophageal varices, liver rupture, hepatic failure, pulmonary embolism and primary peritonitis.

\section{Clinical characteristics}

The mean age of 54 subjects was 57 years (range 32-75) and 47 patients $(87.0 \%)$ were male. Causative liver diseases included chronic hepatitis B (38 patients, $70.3 \%$ ), chronic hepatitis C (6 patients, $11.1 \%$ ) and alcoholic hepatitis (7 patients, $13.0 \%$ ). There were 33 patients (61.1\%) of Child-Turcotte-Pugh class A, 17 patients (31.5\%) of class B and 4 patients $(7.4 \%)$ of class $\mathrm{C}$.

As a result of classifying the subjects according to radiological imaging morphologies of HCC, there were 32 cases of the massive type (59.3\%), 13 cases of the diffused type (24.1\%) and 9 cases of the multinodular type (16.7\%), and there was no single-nodular type [34].

PVTT was also present in 40 patients $(74.1 \%)$. There were 14 patients (25.9\%) of $\mathrm{Vp} 0$ (absence of invasion of (or tumor thrombus in) the portal vein), 6 patients $(16.7 \%)$ of $\mathrm{Vp} 2$ (invasion of (or tumor thrombus in) second order branches of the portal vein), 16 patients (12.5\%) of Vp3 (invasion of (or tumor thrombus in) first order branches of the portal vein) and 18 patients (33.3\%) of $\mathrm{Vp} 4$ (invasion of (or tumor thrombus in) the main trunk of the portal vein and/or contra-lateral portal vein branch to the primarily involved lobe) by the criteria of the Liver Cancer Study Group of Japan [33].

Previous treatment was present in 27 patients (50\%). 24 patients underwent TACE, 2 patients underwent percutaneous ethanol injection and 1 patient underwent surgical operation (Table 1).

\section{Results}

\section{Response to therapy}

The mean numbers of treatment cycle of 54 subjects were 4.2 (2-18 cycles). Among them, as a result of classifying the subjects according to response to therapy by using modified Response Evaluation Criteria in Solid Tumors (mRECIST). After 2 cycles of treatment, there were 0 patients $(0 \%)$ of complete response, 17 patients $(31.5 \%)$ of partial 
response, 17 patients (31.5\%) of stable disease and 20 patients $(37.0 \%)$ of progressive disease.

Among them, 21 patients (38.9\%) showing therapeutic response after 2 cycles of treatment were able to take 4 cycles of treatment. Otherwise, 17 patients who showed no therapeutic response after 2 cycles of treatment did not receive another treatment after HAIC. 3 patients who showed no therapeutic response after 2 cycles of treatment underwent another treatment (radiation therapy or TACE) after HAIC.

After 4 cycles of treatment, there was 1 patient (4.8\%) of complete response, 8 patients $(38.1 \%)$ of partial response, 5 patients $(23.8 \%)$ of stable disease and 7 patients $(33.3 \%)$ of progressive disease. 4 patients who showed no therapeutic response after 4 cycles of treatment

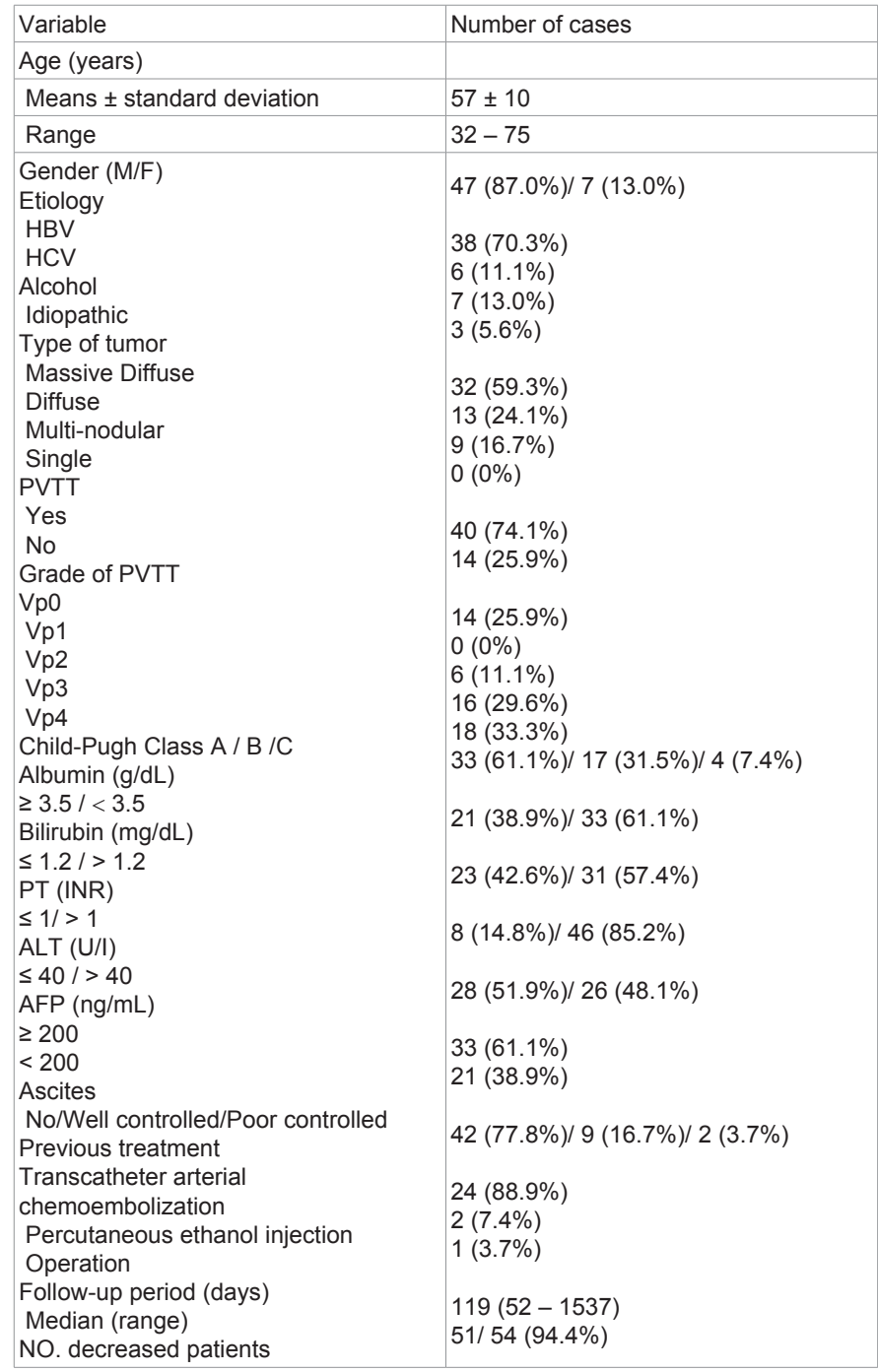

HBV: Hepatitis B Virus; HCV: Hepatitis C Virus; PVTT: Portal Vein Tumor Thrombosis; Vp0: Absence of

invasion of (or tumor thrombus in) the portal vein; Vp1: Invasion (or tumor thrombus in) distal to the second order branches of the portalvein, but not of the second order branches; Vp2: Invasion of (or tumor thrombus in) second order branches of the portal vein; Vp3: Invasion of (or tumor thrombus in) first order branches of the portal vein; Vp4: Invasion of (or tumor thrombus in) the main trunk of the portal veinand/or contra-lateral portal vein branch to the primarily involved lobe; PT: Prothrombin Time; INR: International Normalized Ratio; ALT: Alanine Transaminase: AFP: Alpha-fetoprotein; HAIC: Hepatic Arterial Infusion Chemotherapy; 5-FU: Fluorouracil

Table 1: Baseline Characteristics of Patients.

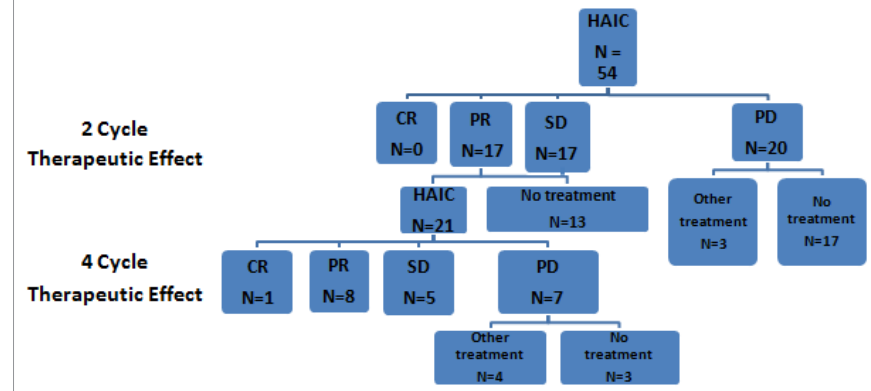

CR: Complete response; PR: Patial response; SD: Stable disease; PD: Progressive disease

Figure 1: Enrollment. HAIC, hepatic artery infusion chemotherapy.

underwent another treatment (radiation therapy or TACE) after HAIC (Figure 1).

Patients with complete response or partial response were classified into the group with therapeutic responses and patients with stable disease or progressive disease were classified into the group having no therapeutic responses by using modified Response Evaluation Criteria in Solid Tumors (mRECIST).

\section{Survival}

The median survival period of 54 patients was 119 days (4.0 months). As a result of analyzing the survival period of the patients treated with the regional HAIC, it was found that while the median survival period of the patient group showing therapeutic response on the abdominal CT image after 2 cycles of treatment was 190 days (6.3 months), that of the patient group showing no therapeutic response was 96 days (3.2 months). This suggested that the therapeutic response may led to a somewhat significant extension of survival period $(\mathrm{p}=$ 0.265) (Figure 2A).

In the case that 4 cycles of treatment or more was conducted, while the median survival period of the patients, who were classified into the patient group with therapeutic responses after 4 cycles of HAIC, was 302 days (10.1 months), that of the patients with no therapeutic responses after 2 cycles or 4 cycles of HAIC was 111 days (3.7 months). Therefore, it showed that the patients with therapeutic responses had a significant extension of survival period $(p=0.009)$ (Figure 2B).

\section{Predictors for response}

Univariate analysis to determine associated factors to the therapeutic responses of the regional HAIC showed that characteristics of the patients (age, $p=0.426$ and sex, $p=0.412$ ) and causative liver disease $(\mathrm{p}=1.000)$ were not associated to the therapeutic responses, and the liver function indexes (Child-Turcotte-Pugh score ( $\mathrm{p}=$ $1.000)$, PT $(p=0.260)$, albumin $(p=0.383)$, Bilirubin $(p=0.769))$, the hematological test (ALT $(p=0.565), \operatorname{APF}(p=0.070)$, the presence of ascites $(p=0.732)$ and previous treatment $(p=0.559)$ also failed to prove any relation to the therapeutic responses.

As a result of analyzing the relation between imaging morphologies of HCC and the therapeutic responses of the regional HAIC after classifying HCC into the massive type, the diffuse type, the multinodular type and the single-nodular type according to CT imaging morphologies of HCC, there was no significant relation between them $(\mathrm{p}=0.246)$. 
A)
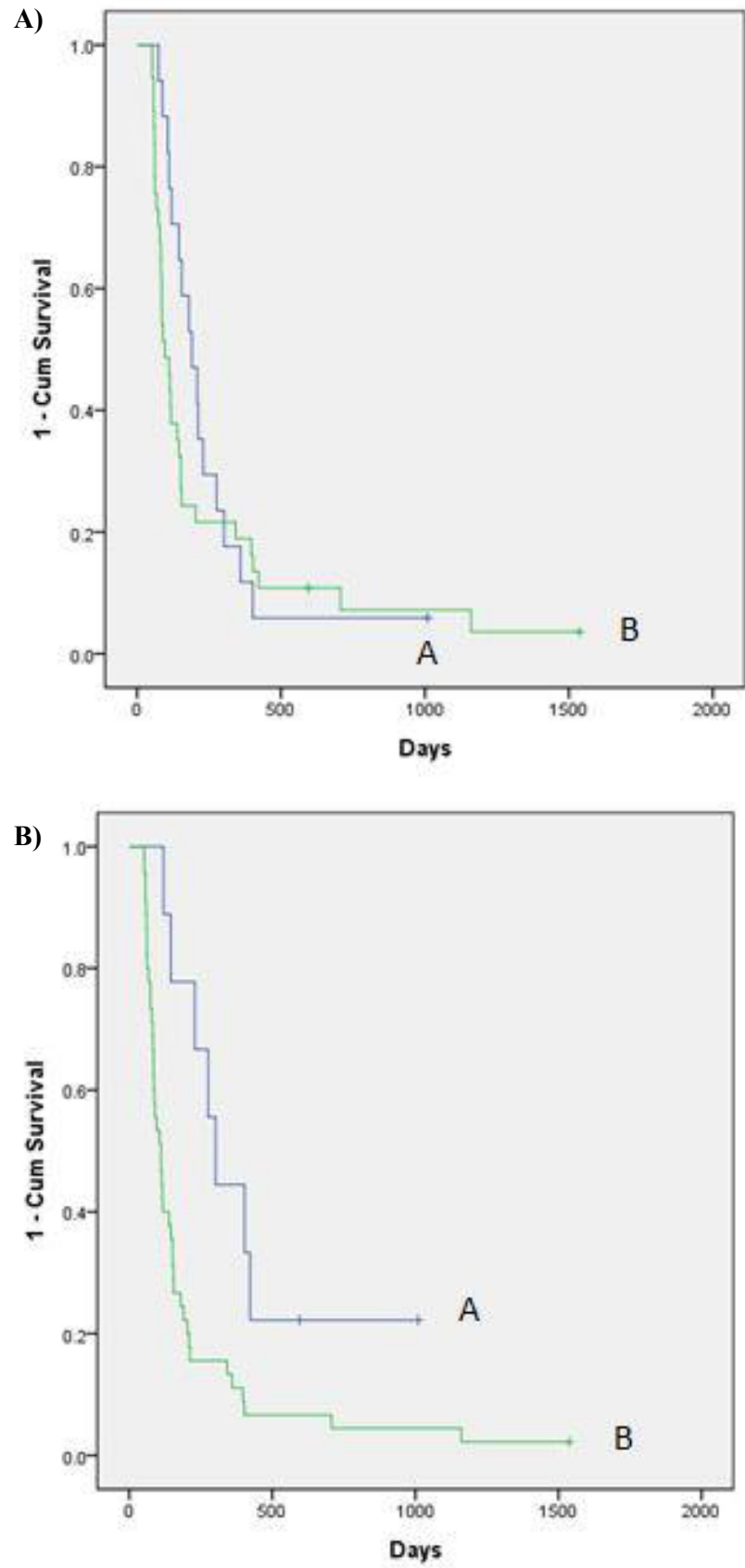

A: Objective Response Group

B: Non-Objective Response Group

Figure 2: Cumulative survival rates according to the responses of advanced $\mathrm{HCC}$ after $2^{\text {nd }}$ cycle and $4^{\text {th }}$ cycle of HAIC. A) Among 54 patients (of whom 17 were in the objective response group after $2^{\text {nd }}$ cycle of HAIC and 37 were in the non-objective response group after $2^{\text {nd }}$ cycle of $\mathrm{HAIC}$ ), the median overall survival was 190 days (6.3 months) in the objective response group, as compared with 96 days (3.2 months) in the non-objective response group ( $p=0.265$ ). B) Among 54 patients (of whom 9 objective response group and 12 non-objective response group after $4^{\text {th }}$ cycle of HAIC and 33 nonobjective response group after $2^{\text {nd }}$ cycle of HAIC), the median overall survival was 302 days (10.1 months) in objective response group, as compared with 111 days $(3.7$ months) in non-objective response group $(p=0.009)$. HAIC: Hepatic Arterial Infusion Chemotherapy.
Meanwhile, as a result of analyzing the relation between the presence of portal vein tumor thrombosis (PVTT) and the therapeutic responses, in patients with no PVTT, the therapeutic response was $57.1 \%$, which was significantly lower than $22.5 \%(\mathrm{p}=0.023)$ in patients with PVTT (Table 2).

Multivariate analysis to determine associated factors to the therapeutic responses of the regional HAIC showed that the presence of PVTT was an independent associated factor to the therapeutic responses of the regional HAIC $(p=0.021)$ (Table 3$)$.

\section{Discussion}

The mortality rate related with liver cirrhosis has been steady or reduced in recent years, and technologic advances in diagnostic

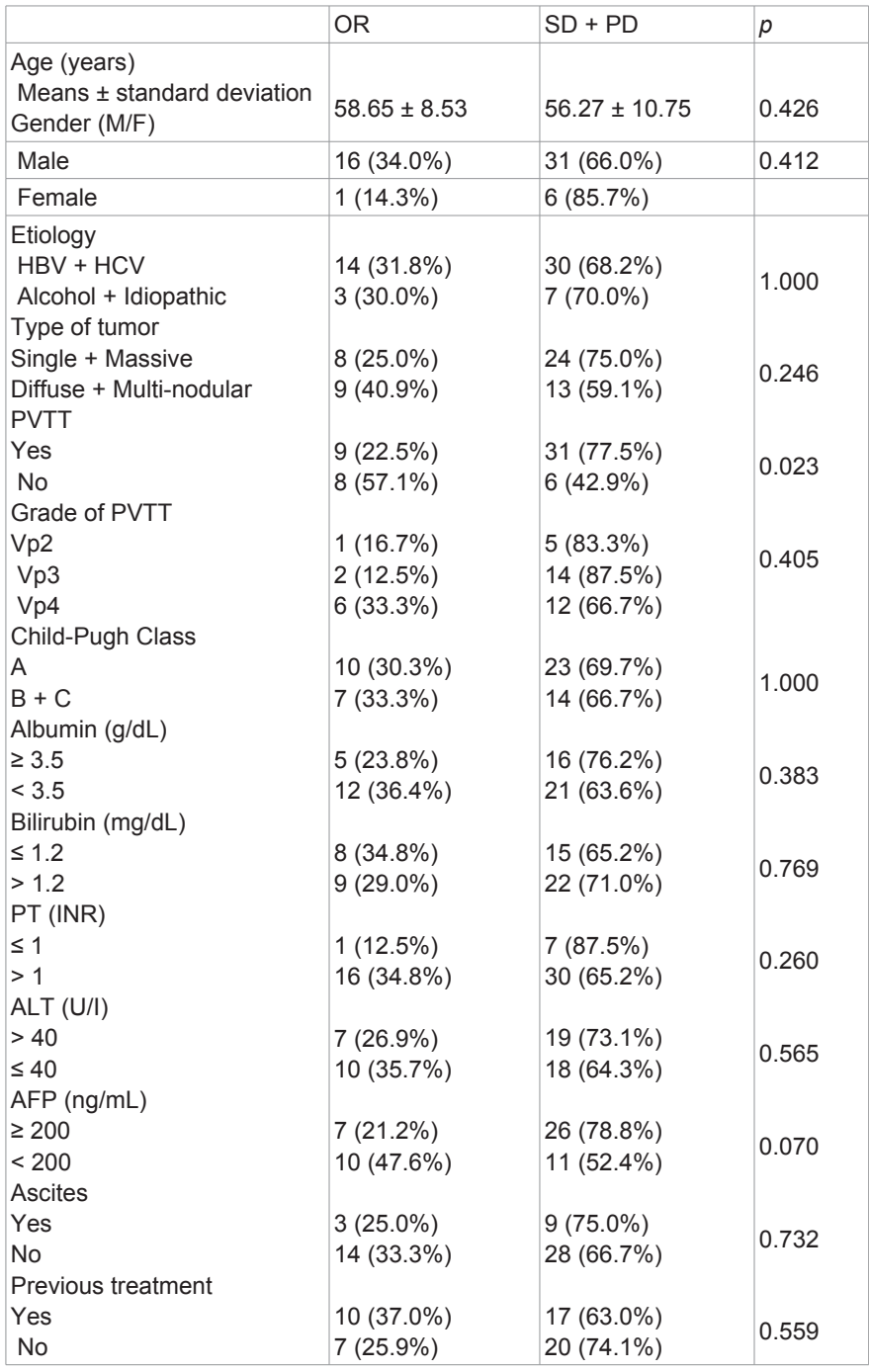

OR: Objective response; SD: Stable disease; PD: Progressive; HBV: Hepatitis B Virus; HCV: Hepatitis C Virus; PVTT: Portal Vein Tumor Thrombosis; Vp1: Invasion (or tumor thrombus in) distal to the second order branches of the portalvein, but not of the second order branches; Vp2: Invasion of (or tumor thrombus in) second order branches of the portal vein; Vp3: Invasionof (or tumor thrombus in) first order branches of the portal vein; Vp4: Invasion of (or tumor thrombus in) the main trunk of the portal veinand/or contra-lateral portal vein branch to the primarily involved lobe; PT: Prothrombin Time; INR: International Normalized Ratio; ALT: Alanine Transaminase: AFP: Alphafetoprotein; HAIC: Hepatic Arterial Infusion Chemotherapy; 5-FU: Fluorouracil

Table 2: Effect of Pre-treatment Variables on the Therapeutic Effect of HAIC in Patients with Advanced HCC according to the Results of Univariate Analysis. 


\begin{tabular}{|l|l|l|l|}
\hline & Odd ratio & $95 \% \mathrm{Cl}$ & $P$ value \\
\hline PVTT & 4.593 & $1.261-16.730$ & 0.021 \\
\hline
\end{tabular}

PVTT: Portal Vein Tumor Thrombosis

Table 3: Effect of Pre-treatment Variables on the Therapeutic Effect of HAIC in Patients with Advanced HCC according to the Results of Multivariate Analysis.

imaging modalities and therapeutic procedures have facilitated early diagnosis and curative treatment in patients with HCC, which is leading to improvement of 5 year survival rate of patients with HCC accompanied with cirrhosis [3-5,35].

Despite this continuous advancement, the prognosis for HCC remains unsatisfactory. The prognosis for HCC at the advanced stage or with PVTT, in particular, remains extremely poor. Okuda et al. [8], reported that the median survival rate of patients with untreated HCC was 0.7 months at Stage 3, 2.0 months in Stage 2 and 8.3 months in Stage 1, and Yeung et al. [11], reported that median survival rate of patients with untreated HCC involving PVTT was 1-2 months. However, it has been reported that even patients with incurable HCC can survive longer if they receive therapeutic procedures $[8,10,11]$.

For this reason, the TACE, the systemic chemotherapy, the radiotherapy or the regional HAIC has been used in patients with advance HCC $[36,37]$. Nevertheless, the HAIC has a potential risk of hepatic failure, and the systemic chemotherapy also has not yet produced satisfactory outcomes [5].

Recent development in the molecular biology makes studies on targeted anti-cancer drugs being actively conducted, and Sorafenib (Nexava ${ }^{\circledR}$; Bayer HealthCare Pharmaceuticals, West Haven, CT, USA) is the most commonly used drug. A significant extension of the survival period was proved in a large scale phase 3 clinical trial (SHARP trial) conducted on 602 patients with HCC, who were treated with Sorafenib, which has been used to treat patients with HCC for whom surgical resection is unavailable under approval by US FDA since November 2007 [38].

Sorafenib has also shown satisfactory drug tolerance, safety and anti-tumor effect in a large scale 3 phase clinical trial (ORIENTAL trial) conducted on 226 patients with HCC in the Asia-Pacific region. In addition, the radiological therapeutic effect [objective response; complete response (CR) and partial reponse (PR)] and the disease control rate following treatment with Sorafenib was reported to be $3.3 \%$ and $35.3 \%$ respectively according to the Barcelona-Clinic Liver Cancer (BCLC) criteria [18,39].

However, the use of Sorafenib is limited due to its adverse effects and its high cost $[19,40]$ and the extension of the survival period in patients in the Asia-Pacific region was reported to be shorter based on comparison of ORIENTAL trial with SHARP trial, which implies there might be racial differences. Furthermore, Kim et al. [20], reported that there wasn't a significant difference between the Sorafenib therapy and the TACE in terms of the survival rate in patients with advanced HCC [20].

The regional HAIC enhance the regional therapeutic effect and decrease adverse reactions, compared with the systemic chemotherapy by injecting the chemotherapeutic agent directly to hepatic artery in consideration of the characteristic of HCC to get blood supplied from hepatic artery, and thereby maintaining the concentration of the chemotherapeutic agent 5-20 times higher therein than in peripheral liver tissue. In addition, it has been reported to be effective in patients with advanced HCC who have difficulties in using other conventional curative treatments have no therapeutic response thereto [21-25].

Recent multi-center prospective studies reported on extension of the survival period and differences in the therapeutic effect depending on the dose of the chemotherapeutic agent in patients with advanced HCC who were treated with the regional HAIC [14,27]. Ueshima et al. [15], and Yamasaki et al. [16], reported that when low-dose 5 -fluorouracil and cisplatin were respectively used in patients with advance HCC, the therapeutic response rates of the regional HAIC were $38.5 \%$ and $38.0 \%$ respectively. Ikeda et al. [17], reported that the therapeutic response rate of the HAIC was $36 \%$ in the group using epirubicin.

Also in this study, the treatment response was defined as the rate of the group presented with complete response (CR) or partial response (PR) among the entire subjects, and the therapeutic response rate after 2 cycles was $31.5 \%$, and after 4 cycles was $42.9 \%$ which was similar to that in other previous studies.

Since many patients with HCC have impaired liver function due to liver cirrhosis, attention should be given to adverse reactions which might be caused by the regional HAIC, and it is, therefore, important to select subjects who could be effectively treated. Woo et al. [27], reported that high-dose of chemotherapeutic agents showed better outcomes than low-dose in their multi-center prospective study conducted on 68 patients using 5-fluorouracil and cisplatin, and that adverse reactions resulting there from were successfully treated as in the case of the low dose chemotherapy.

When it comes to the major associated factor contributing to extension of the survival period by the regional HAIC, Ando et al. [12], reported on extension of the survival period in the group showing therapeutic response, and Kondo et al. [28], reported on extension of the survival period in the group showing therapeutic response, the group aged 70 years or less and the group with low ALT. In addition, Yamasaki et al. [16], reported that the patient group with low AFP and the patient group treated with low-dose of 5-fluorouracil, cisplatin and leucovorin/isovorin showed longer extension of the survival period than the patient group treated with low-dose of cisplatin and 5-FU.

In this study, there was no significant correlation between the characteristics of the subjects [age $(p=0.426)$ and $\operatorname{sex}(p=0.412)$ ], causative liver disease $(\mathrm{p}=1.000)$, liver function index [Child-TurcottePugh score $(\mathrm{p}=1.000)$, PT $(\mathrm{p}=0.260)$, albumin $(\mathrm{p}=0.383)$, Bilirubin $(\mathrm{p}=0.769)]$, hematologic test [ALT $(p=0.565) \operatorname{AFP}(\mathrm{p}=0.070)$, the presence of ascites $(p=0.732)$ and previous treatment $(p=0.559)]$.

In the group showing therapeutic responses, it was found that the survival period was significantly extended $(\mathrm{p}=0.265)$, and in the case of conducting the regional HAIC repetitively, it was found that the survival period was more significantly extended $(p=0.009)$. As a result of taking the abdominal CT scan, it was found that the group showing therapeutic responses survived 3.0 months longer than the group showing no therapeutic responses after 2 cycles of the treatment, and the group showing therapeutic responses after 4 cycles of the treatment survived 6.4 months longer than the group showing no therapeutic responses after 2 cycles or 4 cycles of treatment.

The presence of PVTT was identified as an independent associated factor contributing to the therapeutic responses of the regional HAIC ( $p=0.021)$ and there was no significant difference of the therapeutic responses between the grade of PVTT. It is, therefore, thought that the 
regional HAIC is expected to get better therapeutic effect and longer extension of the survival period in patients with HCC not accompanied with PVTT than in patients with HCC accompanied with PVTT.

In this study, HCC was classified into the massive type (59.3\%), the diffuse type $(24.1 \%)$, the multi-nodular type (16.7\%) and the singlenodular type $(0 \%)$ according to radiological morphologies, and their correlation with therapeutic responses and extension of the survival period was investigated, but there was no relation between treatment responses and radiological morphologies of HCC $(\mathrm{p}=0.246)$.

However, Jang et al. [29], reported the survival period was extended 7.4 month longer in the HCC group without laterally affected extrahepatic vessels or anatomical variations than the HCC group with laterally affected extrahepatic vessels or anatomical variations. So, it will be necessary to conduct further studies on this subject matter.

Since this study was retrospectively conducted, it had some limitations to investigate of associated factors in therapeutic responses and the survival period. It is, therefore, thought that it will be necessary to conduct further prospective studies on a large number of patients and further studies on prognosis factors of the regional HAIC in the future.

In conclusion, it is thought that regional HAIC is expected to get better therapeutic effect and longer extension of survival period in patients with advanced HCC. And, the presence of PVTT was an independent associated factor of therapeutic responses of the regional HAIC.

\section{Acknowledgments}

This investigation was not financially supported.

\section{References}

1. Registry. MoHaWKCC (2008) Cancer incidence in Korea, 1999-2001.

2. Registry. MoHaWKCC (2008) Cancer incidence in Korea, 2007

3. Shin HR, Masuyer E, Ferlay J, Curado MP; Asian Contributors to Cl5 IX4 (2010) Cancer in Asia - Incidence rates based on data in cancer incidence in five continents IX (1998-2002). Asian Pac J Cancer Prev 2: 11-16.

4. Center MM, Jemal A (2011) International trends in liver cancer incidence rates. Cancer Epidemiol Biomarkers Prev 20: 2362-2368.

5. Han KH, Kudo M, Ye SL, Choi JY, Poon RT, et al. (2011) Asian consensus workshop report: expert consensus guideline for the management of intermediate and advanced hepatocellular carcinoma in Asia. Oncology 81 158-164.

6. Thomas MB, O'Beirne JP, Furuse J, Chan AT, Abou-Alfa G, et al. (2008) Systemic therapy for hepatocellular carcinoma: cytotoxic chemotherapy, targeted therapy and immunotherapy. Ann Surg Oncol 15: 1008-1014.

7. Nagasue N, Uchida M, Makino Y, Takemoto Y, Yamanoi A, et al. (1993) Incidence and factors associated with intrahepatic recurrence following resection of hepatocellular carcinoma. Gastroenterology 105: 488-494.

8. Okuda K, Ohtsuki T, Obata H, Tomimatsu M, Okazaki N, et al. (1985) Natural history of hepatocellular carcinoma and prognosis in relation to treatment. Study of 850 patients. Cancer 56: 918-928.

9. Llovet JM, Bustamante J, Castells A, Vilana R, Ayuso Mdel C, et al. (1999) Natural history of untreated nonsurgical hepatocellular carcinoma: rationale for the design and evaluation of therapeutic trials. Hepatology 29: 62-67.

10. Cady B (1983) Natural history of primary and secondary tumors of the liver Semin Oncol 10: 127-134.

11. Yeung YP, Lo CM, Liu CL, Wong BC, Fan ST, et al. (2005) Natural history of untreated nonsurgical hepatocellular carcinoma. Am J Gastroenterol 100: 1995-2004.

12. Ando E, Tanaka M, Yamashita F, Kuromatsu R, Yutani S, et al. (2002) Hepatic arterial infusion chemotherapy for advanced hepatocellular carcinoma with portal vein tumor thrombosis: analysis of 48 cases. Cancer 95: 588-595

13. Park JY, Ahn SH, Yoon YJ, Kim JK, Lee HW, et al. (2007) Repetitive shortcourse hepatic arterial infusion chemotherapy with high-dose 5-fluorouracil and cisplatin in patients with advanced hepatocellular carcinoma. Cancer 110: 129137

14. Kim HY, Kim JD, Bae SH, Park JY, Han KH, et al. (2010) A comparative study of high-dose hepatic arterial infusion chemotherapy and transarterial chemoembolization using doxorubicin for intractable, advanced hepatocellular carcinoma. Korean J Hepatol 16: 355-361.

15. Ueshima K, Kudo M, Takita M, Nagai T, Tatsumi C, et al. (2010) Hepatic arterial infusion chemotherapy using low-dose 5 -fluorouracil and cisplatin for advanced hepatocellular carcinoma. Oncology 78: 148-153.

16. Yamasaki T, Kimura T, Kurokawa F, Aoyama K, Ishikawa T, et al. (2005) Prognostic factors in patients with advanced hepatocellular carcinoma receiving hepatic arterial infusion chemotherapy. J Gastroenterol 40: 70-78.

17. Ikeda M, Okusaka T, Ueno H, Morizane C, Iwasa S, et al. (2007) Hepatic arteria infusion chemotherapy with epirubicin in patients with advanced hepatocellular carcinoma and portal vein tumor thrombosis. Oncology 72: 188-193.

18. Cheng AL, Kang YK, Chen Z, Tsao CJ, Qin S, et al. (2009) Efficacy and safety of sorafenib in patients in the Asia-Pacific region with advanced hepatocellular carcinoma: a phase III randomised, double-blind, placebo-controlled trial. Lancet Oncol 10: 25-34.

19. Lee JM, Han KH (2010) Positioning and indication of sorafenib in the treatmen algorithm and real practice setting: Western and eastern approach--Asian perspective. Oncology 78: 167-171

20. Kim HY, Park JW, Nam BH, Kim HK, Choi Jl, et al. (2011) Survival of patients with advanced hepatocellular carcinoma: sorafenib versus other treatments. J Gastroenterol Hepatol 26: 1612-1618.

21. Yamasaki T, Sakaida I (2012) Hepatic arterial infusion chemotherapy for advanced hepatocellular carcinoma and future treatments for the poor responders. Hepatol Res 42: 340-348.

22. Murakami E, Aikata H, Miyaki D, Nagaoki Y, Katamura Y, et al. (2012) Hepatic arterial infusion chemotherapy using 5-fluorouracil and systemic interferon- $\alpha$ for advanced hepatocellular carcinoma in combination with or without threedimensional conformal radiotherapy to venous tumor thrombosis in hepatic vein or inferior vena cava. Hepatol Res 42: 442-453.

23. Jeong SW, Jang JY, Lee JE, Lee SH, Kim SG, et al. (2012) The efficacy of hepatic arterial infusion chemotherapy as an alternative to sorafenib in advanced hepatocellular carcinoma. Asia Pac J Clin Oncol 8: 164-171.

24. Kim BK, Park JY, Choi HJ, Kim do Y, Ahn SH, et al. (2011) Long-term clinica outcomes of hepatic arterial infusion chemotherapy with cisplatin with or without 5-fluorouracil in locally advanced hepatocellular carcinoma. J Cancer Res Clin Oncol 137: 659-667.

25. Niizeki T, Sumie S, Torimura T, Kurogi J, Kuromatsu R, et al. (2012) Serum vascular endothelial growth factor as a predictor of response and survival in patients with advanced hepatocellular carcinoma undergoing hepatic arterial infusion chemotherapy. J Gastroenterol 47: 686-695

26. Korean Liver Cancer Study Group and National Cancer Center, Korea (2009) Practice guidelines for management of hepatocellular carcinoma 2009. Korean J Hepatol 15: 391-423.

27. Woo HY, Bae SH, Park JY, Han KH, Chun HJ, et al. (2010) A randomized comparative study of high-dose and low-dose hepatic arterial infusion chemotherapy for intractable, advanced hepatocellular carcinoma. Cancer Chemother Pharmacol 65: 373-382.

28. Kondo M, Morimoto M, Numata K, Nozaki A, Tanaka K (2011) Hepatic arteria infusion therapy with a fine powder formulation of cisplatin for advanced hepatocellular carcinoma with portal vein tumor thrombosis. Jpn J Clin Oncol 41: $69-75$.

29. Jang BK, Chung WJ, Park KS, Cho KB, Hwang JS, et al. (2005) The efficacy of hepatic arterial infusion therapy for advanced hepatocellular carcinoma according to extrahepatic collateral feeding vessels. Korean J Hepatol 11: 359 370

30. Trevisani F, D'Intino PE, Morselli-Labate AM, Mazzella G, Accogli E, et al (2001) Serum alpha-fetoprotein for diagnosis of hepatocellular carcinoma in 
Citation: Choi JH, Chung WJ, Jang BK, Hwang JS (2012) Survival and Treatment Responses of Hepatic Arterial Infusion Chemotherapy for Advanced Hepatocellular Carcinoma. J Cancer Sci Ther 4: 292-298. doi:10.4172/1948-5956.1000157

patients with chronic liver disease: influence of HBsAg and anti-HCV status. J Hepatol 34: 570-575.

31. Eisenhauer EA, Therasse P, Bogaerts J, Schwartz LH, Sargent D, et al. (2009) New response evaluation criteria in solid tumours: revised RECIST guideline (version 1.1). Eur J Cancer 45: 228-247.

32. Edeline J, Boucher E, Rolland Y, Vauléon E, Pracht M, et al. (2012) Comparison of tumor response by Response Evaluation Criteria in Solid Tumors (RECIST) and modified RECIST in patients treated with sorafenib for hepatocellular carcinoma. Cancer 118: 147-156.

33. (1989) The general rules for the clinical and pathological study of primary liver cancer. Liver Cancer Study Group of Japan. Jpn J Surg 19: 98-129.

34. Yuki K, Hirohashi S, Sakamoto M, Kanai T, Shimosato Y (1990) Growth and spread of hepatocellular carcinoma. A review of 240 consecutive autopsy cases. Cancer 66: 2174-2179.

35. Fattovich G, Stroffolini T, Zagni I, Donato F (2004) Hepatocellular carcinoma in cirrhosis: incidence and risk factors. Gastroenterology 127: S35-S50.
36. Kang MK, Kim MS, Kim SK, Ye GW, Lee HJ, et al. (2011) High-dose radiotherapy with intensity-modulated radiation therapy for advanced hepatocellular carcinoma. Tumori 97: 724-731.

37. Rampone B, Schiavone B, Martino A, Viviano C, Confuorto G (2009) Current management strategy of hepatocellular carcinoma. World J Gastroenterol 15: 3210-3216.

38. Llovet JM, Ricci S, Mazzaferro V, Hilgard P, Gane E, et al. (2008) Sorafenib in advanced hepatocellular carcinoma. N Engl J Med 359: 378-390.

39. Yau T, Chan P, Ng KK, Chok SH, Cheung TT, et al. (2009) Phase 2 open-label study of single-agent sorafenib in treating advanced hepatocellular carcinoma in a hepatitis B-endemic Asian population: presence of lung metastasis predicts poor response. Cancer 115: 428-436.

40. Ogasawara S, Kanai F, Obi S, Sato S, Yamaguchi T, et al. (2011) Safety and tolerance of sorafenib in Japanese patients with advanced hepatocellular carcinoma. Hepatol Int 5: 850-856. 